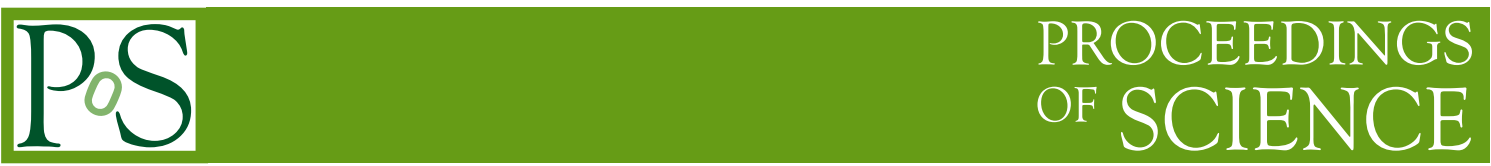

\title{
Leptogenesis in neutrinophilic Higgs doublet models
}

\section{Naoyuki Haba}

Department of Physics, Osaka University, Toyonaka, Osaka 560-0043, Japan

E-mail: haba@phys.sci.osaka-u.ac.jp

\section{Osamu Seto*}

Department of Architecture and Building Engineering, Hokkai-Gakuen University, Sapporo 062-8605, Japan

E-mail: seto@physics.umn.edu

In a class of two Higgs doublet model, where one Higgs doublet generates masses of quarks and charged leptons whereas the other Higgs doublet with a tiny vacuum expectation value(VEV) generates neutrino Dirac masses, thermal leptogenesis suitably works at low energy scale as keeping no enhancement of lepton number violating washout effects. In addition, Thermal leptogenesis in a supersymmetric neutrinophilic Higgs model also similarly works. The mass of the lightest righthanded (s)neutrino can be reduced to be $\mathscr{O}\left(10^{5}\right) \mathrm{GeV}$ as keeping sufficiently large CP asymmetry in its decay. Therefore, the reheating temperature after inflation is not necessarily high, hence this scenario is free from gravitino problem.

The 2011 Europhysics Conference on High Energy Physics-HEP 2011,

July 21-27, 2011

Grenoble, Rhône-Alpes France

${ }^{*}$ Speaker. 


\section{Introduction}

Thermal leptogenesis [1] by heavy right-handed Majorana neutrinos for seesaw mechanism [2] is one of the most attractive scenarios for baryogeneis. The size of $\mathrm{CP}$ asymmetry in a right-handed neutrino decay is, roughly speaking, proportional to the mass of right-handed neutrino. It has been regarded that leptogenesis in low energy scale is difficult in general [3, 4].

A new class of two Higgs doublet models (THDM) called "neutrinophilic Higgs doublet model" has been considered [5, 6, 7, 8, 9, 10, 11, 12, 13]. The idea of this class of models is that neutrino masses are much smaller than other fermions because those come from the different Higgs doublet with a smaller VEV.

Notice that neutrino Yukawa couplings in neutrinophilic Higgs doublet models do not need to be so small for lighter right-handed neutrinos. This fact has significant implication to leptogenesis. This opens new possibility of low scale thermal leptogenesis. Here, we will show that $\mathrm{CP}$ asymmetry is enhanced and thermal leptogenesis suitably works in multi-Higgs models with a neutrinophilic Higgs doublet field [14]. The realization of low scale thermal leptogenesis is attractive particularly in supersymmetric models, because of "gravitino problem" [15]. Hence, thermal leptogenesis at a low energy scale in a neutrinophilic Higgs doublet model offers a new solution to avoid gravitino problem $[14,16]$.

\section{Minimal neutrinophilic THDM}

Let us show the minimal neutrinophilic THDM model originally suggested by Ma [5]. In the model, one additional Higgs doublet $\Phi_{v}$, which gives only neutrino Dirac masses, besides the SM Higgs doublet $\Phi$ and a discrete $Z_{2}$-parity are introduced. Under the discrete symmetry, Yukawa interactions are given by

$$
\mathscr{L}_{\text {yukawa }}=y^{u} \bar{Q}_{L} \Phi U_{R}+y^{d} \bar{Q}_{L} \tilde{\Phi} D_{R}+y^{l} \bar{L} \Phi E_{R}+y^{v} \bar{L} \Phi_{v} N+\frac{1}{2} M \bar{N}^{c} N+\text { h.c. }
$$

where $\tilde{\Phi}=i \sigma_{2} \Phi^{*}$, and we omit a generation index. $\Phi_{v}$ only couples with a right-handed neutrino $N$ by the $Z_{2}$-parity so that flavor changing neutral currents (FCNCs) are suppressed. The Higgs potential of the neutrinophilic THDM is given by

$$
\begin{aligned}
V^{\mathrm{THDM}}=m_{\Phi}^{2} \Phi^{\dagger} \Phi+m_{\Phi_{v}}^{2} \Phi_{v}^{\dagger} \Phi_{v}-m_{3}^{2}\left(\Phi^{\dagger} \Phi_{v}+\Phi_{v}^{\dagger} \Phi\right)+\frac{\lambda_{1}}{2}\left(\Phi^{\dagger} \Phi\right)^{2}+\frac{\lambda_{2}}{2}\left(\Phi_{v}^{\dagger} \Phi_{v}\right)^{2} \\
+\lambda_{3}\left(\Phi^{\dagger} \Phi\right)\left(\Phi_{v}^{\dagger} \Phi_{v}\right)+\lambda_{4}\left(\Phi^{\dagger} \Phi_{v}\right)\left(\Phi_{v}^{\dagger} \Phi\right)+\frac{\lambda_{5}}{2}\left[\left(\Phi^{\dagger} \Phi_{v}\right)^{2}+\left(\Phi_{v}^{\dagger} \Phi\right)^{2}\right] .
\end{aligned}
$$

The $Z_{2}$-symmetry is softly broken by $m_{3}^{2}$.

\section{Leptogenesis in neutrinophilic THDM}

We consider leptogenesis in the neutrinophilic THDM with the extra Higgs doublet $\Phi_{v}$ described in Sec. 2. Under hierarchical right-handed neutrino mass spectrum, the CP asymmetry,

$$
\varepsilon \simeq-\frac{3}{16 \pi} 10^{-6}\left(\frac{0.1 \mathrm{GeV}}{v_{v}}\right)^{2}\left(\frac{M_{1}}{100 \mathrm{GeV}}\right)\left(\frac{m_{v}}{0.05 \mathrm{eV}}\right) \sin \delta,
$$




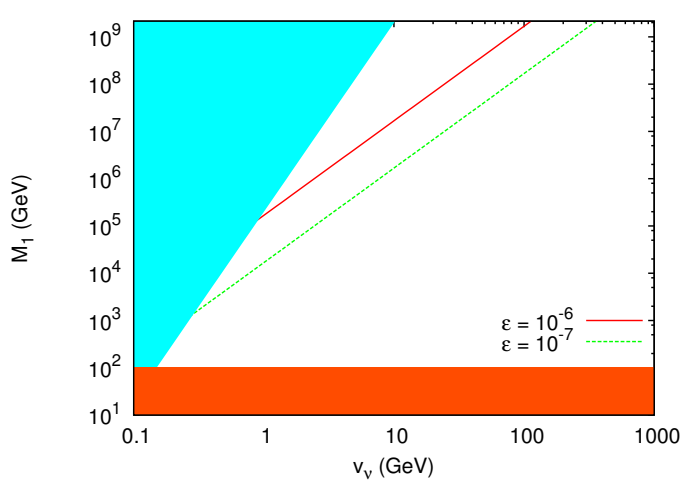

Figure 1: Available region for leptogenesis [14]. The horizontal axis is the VEV of neutrino Higgs $v_{v}$ and the vertical axis is the mass of the lightest right- Figure 2: Available region for leptogenesis in a suhanded neutrino mass $M_{1}$. In the red brown region, the persymmetric neutrinophilic model [16]. The blue lightest right-handed neutrino decay is kinematically dashed line denotes a reference value for the upper forbidden. In turquoise region, $\Delta L=2$ washout effect bound [17] of reheating temperature after inflation $T_{R}$. is too strong. The red and green line are contours of the CP asymmetry of $\varepsilon=10^{-6}$ and $10^{-7}$, respectively.

is significantly enhanced for a light right-handed neutrinos due to the tiny Higgs VEV, $v_{v}$. On the other hand, one should notice that, for lower $v_{v}$, the $\Delta L=2$ lepton number violating washout processes become more significant.

All conditions for successful thermal leptogenesis is presented in Fig. 1. The horizontal axis is the VEV of neutrino Higgs $v_{v}$ and the vertical axis is the mass of the lightest right-handed neutrino, $M_{1}$. In the red brown region, the out of equilibrium decay of lightest right-handed neutrino is not possible. In turquoise region, $\Delta L=2$ lepton number violating washout effect is too strong. The red and green line are contours of the CP asymmetry of $\varepsilon=10^{-6}$ and $10^{-7}$, respectively. Thus, in the parameter region above the line of $\varepsilon=10^{-7}$, thermal leptogenesis easily works even with hierarchical masses of right-handed neutrinos.

\section{Leptogenesis in a supersymmetric neutinophilc model}

Construct a supersymmetric model with $\Phi_{v}$ is straintforword $[14,16]$. By repeating the same analysis for leptogenesis in the previous section, we can find the availability of thermal leptogenesis and its resuts are summserised in Fig. 2. A sufficient CP violation $\varepsilon=\mathscr{O}\left(10^{-6}\right)$ can be realized for $v_{v}=\mathscr{O}(1) \mathrm{GeV}$ in the hierarchical right-handed neutrino with $M_{1}$ of $\mathscr{O}\left(10^{5}-10^{6}\right) \mathrm{GeV}$. This implies that the reheating temperature after inflation $T_{R}$ of $\mathscr{O}\left(10^{6}\right) \mathrm{GeV}$ is high enough to produce right-handed neutrinos by thermal scatterings. Thus, this class of model with $v_{v}=\mathscr{O}(1) \mathrm{GeV}$ is a solution to compatible with thermal leptogenesis in gravity mediated supersymmetry breaking with unstable gravitino.

\section{Conclusion}

We have examined the possibility of thermal leptogenesis in neutrinophilic Higgs doublet 
models. We have found the available parameter region of thermal leptogenesis where its washout effect is avoided as keeping the $\mathrm{CP}$ asymmetry large enough. In addition, in a supersymmetric neutrinophilic Higgs doublet model, we have pointed out that thermal leptogenesis in gravity mediated SUSY breaking works well by a relatively light right-handed neutrino with the mass of $\mathscr{O}\left(10^{5}\right)$ $\mathrm{GeV}$ without confronting gravitino problem.

\section{Acknowledgements}

This work is partially supported by Scientific Grant by Ministry of Education and Science, Nos. 20540272, 22011005, 20244028, and 21244036 (N.H.), and the scientific research grants from Hokkai-Gakuen (O.S.).

\section{References}

[1] M. Fukugita and T. Yanagida, Phys. Lett. B 174, 45 (1986).

[2] P. Minkowski, Phys. Lett. B 67, 421 (1977);

T. Yanagida, in Proceedings of Workshop on the Unified Theory and the Baryon Number in the Universe, Tsukuba, Japan, edited by A. Sawada and A. Sugamoto (KEK, Tsukuba, 1979), p 95; M. Gell-Mann, P. Ramond, and R. Slansky, in Supergravity, Proceedings of Workshop, Stony Brook, New York, 1979, edited by P. Van Nieuwenhuizen and D. Z. Freedman (North-Holland, Amsterdam, 1979), p 315.

[3] W. Buchmuller, P. Di Bari and M. Plumacher, Nucl Phys B 643, 367 (2002).

[4] S. Davidson and A. Ibarra, Phys. Lett. B 535, 25 (2002).

[5] E. Ma, Phys. Rev. Lett. 86, 2502 (2001).

[6] F. Wang, W. Wang and J. M. Yang, Europhys. Lett. 76, 388 (2006).

[7] S. Gabriel and S. Nandi, Phys. Lett. B 655, 141 (2007).

[8] E. Ma, Phys. Rev. D 73, 077301 (2006).

[9] S. M. Davidson and H. E. Logan, Phys. Rev. D 80, 095008 (2009).

[10] H. E. Logan and D. MacLennan, Phys. Rev. D 81, 075016 (2010).

[11] N. Haba and M. Hirotsu, Eur. Phys. J. C 69, 481 (2010).

[12] N. Haba and K. Tsumura, JHEP 1106, 068 (2011).

[13] N. Haba and T. Horita, Phys. Lett. B 705, 98 (2011).

[14] N. Haba and O. Seto, Prog. Theor. Phys. 125, 1155 (2011).

[15] M. Y. Khlopov and A. D. Linde, Phys. Lett. B 138, 265 (1984);

J. R. Ellis, J. E. Kim and D. V. Nanopoulos, Phys. Lett. B 145, 181 (1984).

[16] N. Haba and O. Seto, Phys. Rev. D 84, 103524 (2011).

[17] For recent analysis, see e.g.,

M. Kawasaki, K. Kohri, T. Moroi and A. Yotsuyanagi, Phys. Rev. D 78, 065011 (2008);

R. H. Cyburt, J. Ellis, B. D. Fields, F. Luo, K. A. Olive and V. C. Spanos, JCAP 0910, 021 (2009). 